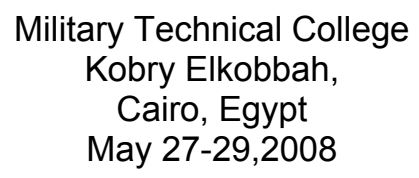

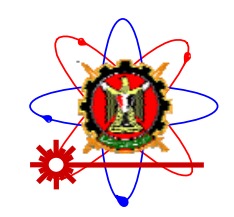

\title{
Numerical Solution of Maxwell's Equations Two Time-Level Difference Scheme TTLD
}

\author{
$4^{\text {th }}$ International Conference on \\ Mathematics and Engineering \\ Physics (ICMEP-4)
}

\author{
F. Hassan
}

\section{Abstract:}

This paper deals with numerical approximations of electromagnetic phenomena, which described by Maxwell's equations,. A two time-level difference scheme (TTLD) is introduced for solving Maxwell's equation as wave equation; this is to reduce the large computational and storage costs of Yee scheme. Convergence and stability conditions have been studied. The comparison with Yee scheme is presented.

\section{1- Introduction}

Maxwell's equations have many important implications in the life of a modern person. The principles of electromagnetism have been deduced from experimental observations. These principles are faraday's law, Ampere's law and Gauss's laws for electric and magnetic fields. These equations had appeared throughout James Clerk Maxwell's 1861. Those equations describe the interrelationship between electric field, magnetic field, electric charge, and electric current, [5] and [13]. This alteration in Ampere's law provides that a changing electric flux produces a magnetic field, just as Faraday's law provides that a changing magnetic field produces an electric field. The relationships of electricity and magnetism are called Maxwell's equations, [10].

The exact solution of Maxwell's system is very complicated or even impossible; this is why numerical methods are generally applied. The first and still applied method is the Finite Difference Time Domain (FDTD) constructed by K. Yee in 1966. Despite the simplicity of the scheme, it requires large computational and storage costs, [1] and [3].

\section{2- Maxwell's Equations}

Let $\Omega \times \mathrm{T}$ be the Cartesian product of a bound simply-connected domain $\Omega$ and a non- negative time interval T. Let $\Omega$, have a smooth or a polygonal boundary $\Gamma$. Electromagnetic phenomenon in $\Omega \times \mathrm{T}$ can described by the differential equations

$$
\begin{aligned}
& \nabla \times E=-\frac{\partial B}{\partial t} \quad \text { In } \quad \Omega \times T \\
& \nabla \times H=\frac{\partial D}{\partial t}+j \quad \text { In } \quad \Omega \times T
\end{aligned}
$$

With the linear material; constitutive relations

$$
\left\{\begin{aligned}
D & =\in E \\
B & =\mu H \\
j & =\sigma E
\end{aligned} \quad \text { In } \quad \Omega \times T\right.
$$

Coupled with Gauss's law

$$
\begin{aligned}
& \nabla \cdot B=0 \\
& \nabla \cdot D=\rho
\end{aligned}
$$

Equation (1) and equation (2) are Faraday and Amper's laws of Maxwell's equations. E is the electric field, $\mathrm{H}$ is the magnetic field, $\mathrm{J}$ is the total electric current density, $\mu$ is the magnetic permeability, we assume that 
the magnetic permeability does not depend on time and $E$ is the electric permittivity, in vacuum we have $c=1 / \sqrt{\mu_{0} \in_{0}}$. The electric permittivity is also assumed not depend on time, $\sigma$ is the electric conductivity, its value is non-negative for dissipative structures [1], [3], [10], [11], [12] and [13]. Both E and $\mathrm{H}$ are vectors in three dimensions, and then equation (1) and equation (2) can be written as

$$
\begin{aligned}
& \left(\begin{array}{c}
\frac{\partial E_{x}}{\partial t} \\
\frac{\partial E_{y}}{\partial t} \\
\frac{\partial E_{z}}{\partial t}
\end{array}\right)=\frac{1}{\epsilon}\left(\begin{array}{l}
\frac{\partial H_{z}}{\partial y}-\frac{\partial H_{y}}{\partial z} \\
\frac{\partial H_{x}}{\partial z}-\frac{\partial H_{z}}{\partial x} \\
\frac{\partial H_{y}}{\partial x}-\frac{\partial H_{x}}{\partial y}
\end{array}\right)-\sigma\left(\begin{array}{c}
E_{x} \\
E_{y} \\
E_{z}
\end{array}\right) \\
& \left(\begin{array}{l}
\frac{\partial H_{x}}{\partial t} \\
\frac{\partial H_{y}}{\partial t} \\
\frac{\partial H_{z}}{\partial t}
\end{array}\right)=-\frac{1}{\mu}\left(\begin{array}{l}
\frac{\partial E_{z}}{\partial y}-\frac{\partial E_{y}}{\partial z} \\
\frac{\partial E_{x}}{\partial z}-\frac{\partial E_{z}}{\partial x} \\
\frac{\partial E_{y}}{\partial x}-\frac{\partial E_{x}}{\partial y}
\end{array}\right)
\end{aligned}
$$

Since the electric field and magnetic field travel perpendicular to one another, thus their dot product must be zero. The solution of Maxwell's equations means the computation of the field strengths using the material parameters and some initial and boundary conditions. For simplicity we suppose that there are no conductive currents and free charges in the computational domain, thus we must solve only the system consisting of equation (1) and equation (2) without electric conductive current density, [7]. The magnetic field well be constrained to two dimensional $(x-y)$-plane. The electric field is then constrained to the $z$ direction. Hence equation (5) and equation (6) become

$$
\begin{aligned}
& \in \frac{\partial E_{z}}{\partial t}=\frac{\partial H_{y}}{\partial x}-\frac{\partial H_{x}}{\partial y} \\
& \mu \frac{\partial H_{x}}{\partial t}=-\frac{\partial E_{z}}{\partial y} \\
& \mu \frac{\partial H_{y}}{\partial t}=\frac{\partial E_{z}}{\partial x}
\end{aligned}
$$

The above set of equations usually referred to as the transverse magnetic (TM) mode [10]. Most (FDTD) scheme solves the time-dependent Maxwell equations using algorithms based on Yee scheme. A limitation of Yee scheme techniques is that their stability is conditional, [7].

\section{3-Finite-Difference Time-Domain Method (FDTD)}

The time-dependent Maxwell's equations (in partial differential form) are discredited using centraldifference approximations to the space and time partial derivatives. The resulting finite-difference equations are solved in a leapfrog manner. The electric field vector components in a volume of space are solved at a given instant in time, then the magnetic field vector components in the same spatial volume are solved at the next instant in time; and the process is repeated over and over again until stop. [1], [3], [9], [10], [13], and [14]. 


\section{Two Time-Level Finite Difference Scheme (TTLD)}

In this paper, Maxwell's equations are solved using a simple finite difference scheme without using leapfrog manner. The solution is computed as follows.

1. Elimination of $\mathrm{H}$ (or E) in equation (1) and equation (2) to obtain the wave equation (This is to reduce the large computational and storage costs of Yee scheme)

2. Determine a finite difference scheme which will be used to solve the reduced set of equations.

3. Studying convergence, consistency and stability for the proposed scheme.

\subsection{The Wave Equation}

4. If we take the time derivative of Faraday and Ampere's in Maxwell's equation and assume that the material properties are time independent, we obtain

$$
\begin{aligned}
& \in \frac{\partial^{2} E_{z}}{\partial t^{2}}=\frac{\partial}{\partial x} \frac{\partial H_{y}}{\partial t}-\frac{\partial}{\partial y} \frac{\partial H_{x}}{\partial t} \\
& \in \frac{\partial^{2} E_{z}}{\partial t^{2}}=\frac{\partial}{\partial x}\left(\frac{1}{\mu} \frac{\partial E_{z}}{\partial x}\right)-\frac{\partial}{\partial y}\left(-\frac{1}{\mu} \frac{\partial E_{z}}{\partial y}\right)
\end{aligned}
$$

This reduces to

$$
\frac{\partial^{2} E_{z}}{\partial t^{2}}=c^{2}\left(\frac{\partial^{2} E_{z}}{\partial x^{2}}+\frac{\partial^{2} E_{z}}{\partial y^{2}}\right)
$$

Where $c=1 / \sqrt{\mu \in}$ is the speed of propagation for the electromagnetic wave. In a similar manner,

$$
\begin{aligned}
& \mu \frac{\partial}{\partial t} \frac{\partial H_{x}}{\partial t}=-\frac{\partial}{\partial y} \frac{\partial E_{z}}{\partial t} \\
& \frac{\partial^{2} H_{x}}{\partial t^{2}}=c^{2}\left(\frac{\partial^{2} H_{x}}{\partial y^{2}}-\frac{\partial^{2} H_{y}}{\partial y \partial x}\right)
\end{aligned}
$$

by the same way

$$
\frac{\partial^{2} H_{y}}{\partial t^{2}}=c^{2}\left(\frac{\partial^{2} H_{y}}{\partial x^{2}}-\frac{\partial^{2} H_{x}}{\partial y \partial x}\right)
$$

Now, equation (10) is used to find approximate solution for electric field in $z$ direction. Equations (11) and (12) are used together to find an approximate solution of the magnetic field. By using equation (10) we can find approximate solution of $E_{z}$ at a new time if we know the values of $E_{z}$ at the boundary, and $E_{z}$ and $\frac{\partial E_{z}}{\partial t}$ at the initial time. By using the central difference approximation in both side of equation (10), we may show that

$$
\begin{aligned}
\left(E_{z}\right)_{i, j}^{k+1}= & r\left[\left(E_{z}\right)_{i+1, j}^{k}+\left(E_{z}\right)_{i-1, j}^{k}\right]+(2-4 r)\left(E_{z}\right)_{i, j}^{k} \\
& +r\left[\left(E_{z}\right)_{i, j+1}^{k}+\left(E_{z}\right)_{i, j-1}^{k}\right]-\left(E_{z}\right)_{i, j}^{k-1}
\end{aligned}
$$

Where $r=\left(\frac{\Delta t}{h} c\right)^{2}=\frac{\Delta t^{2}}{h^{2} \in \mu}, \quad \Delta x=\Delta y=h$. Equation (13) gives a formula for the unknown electric field $\left(E_{z}\right)_{i, j}^{k+1}$ at $(i, j, k+1)^{t h}$ mesh point in terms of known electric field along the $k^{t h}$ and $(k-1)^{\text {th }}$ time rows. 


\section{Analysis study}

This section is concerned with the conditions that must be satisfied if the solution of the finite-difference equations is to be reasonably accurate approximation to the solution of the corresponding partial differential equation.

\subsubsection{The Stability of TTLD Scheme}

The vector solution $E^{k+1}$ of the finite difference equations at the $(k+1)^{t h}$ time-level is related to the vectors solution $E^{k}$ and $E^{k-1}$ at the $k^{t h}$ and $(k-1)^{t h}$ time-levels respectively by the following equation.

$$
\left(\begin{array}{c}
E_{i, 1}^{k+1} \\
\vdots \\
E_{i, j}^{k+1} \\
\vdots \\
E_{i, N-1}^{k+1}
\end{array}\right)=\left(\begin{array}{ccccc}
D & R & & & \\
& \ddots & \ddots & & \\
& R & D & R & \\
& & \ddots & \ddots & \\
& & & R & D
\end{array}\right)\left(\begin{array}{c}
E_{i, 1}^{k} \\
\vdots \\
E_{i, j}^{k} \\
\vdots \\
E_{i, N-1}^{k}
\end{array}\right)-\left(\begin{array}{c}
E_{i, 1}^{k-1} \\
\vdots \\
E_{i, j}^{k-1} \\
\vdots \\
E_{i, N-1}^{k-1}
\end{array}\right)+\left(\begin{array}{c}
C_{i, 1}^{k} \\
\vdots \\
C_{i, j}^{k} \\
\vdots \\
C_{i, N-1}^{k}
\end{array}\right)
$$

where $C$ is a vector of known values, $i=1, . ., N-1, j=1, . ., N-1$ and $k=1,2, . ., T$. equation (14) can be written as

$$
E^{k+1}=A E^{k}-E^{k-1}+C^{k}
$$

Where $A$ is $(N-1)^{2} \times(N-1)^{2}$ blocktridiagonal matrix as is displayed. Each $D$ and $R$ are $(N-1) \times(N-1)$ matrix have the following form

$$
D=\left(\begin{array}{ccccc}
(2-4 r) & r & & & \\
& \ddots & \ddots & & \\
& r & (2-4 r) & r & \\
& & \ddots & \ddots & \\
& & & r & (2-4 r)
\end{array}\right)
$$

$$
R=r I
$$

Equation(13) can be written as

$$
\left[\begin{array}{c}
E^{k+1} \\
E^{k}
\end{array}\right]=\left[\begin{array}{cc}
A & -I \\
I & O
\end{array}\right]\left[\begin{array}{c}
E^{k} \\
E^{k-1}
\end{array}\right]+\left[\begin{array}{c}
C^{k} \\
O
\end{array}\right]
$$

where $I$ is the unit matrix of order $(N-1)^{2}$, This technique has reduced a three-level difference equation to two-level one, [2] and [4].

$$
P=\left[\begin{array}{cc}
A & -I \\
I & O
\end{array}\right]
$$

Equation (16) will be stable when each Eigen value of $P$ has a modules $\leq 1$. The matrix $A$ has $(N-1)^{2}$ different eigenvalus. Also the matrix $I$ has $(N-1)^{2}$ eigenvalus each equal 1 . Hence the eigenvalues $\lambda$ of $P$ are the eigenvalus of the matrix

$$
\left[\begin{array}{cc}
\lambda_{s} & -1 \\
1 & 0
\end{array}\right]
$$

Where $\lambda_{s}$ is the $s^{t h}$ Eigen value of $A$. The eigenvalus of $A$ are given by the eigenvalus of matrices 


$$
\left(\begin{array}{ccccc}
\lambda_{D}^{(s)} & \lambda_{R}^{(s)} & & & \\
& \ddots & \ddots & & \\
& \lambda_{R}^{(s)} & \lambda_{D}^{(s)} & \lambda_{R}^{(s)} & \\
& & \ddots & \ddots & \\
& & & \lambda_{R}^{(s)} & \lambda_{D}^{(s)}
\end{array}\right)
$$

Where $\lambda_{D}^{(s)}$ are the $s^{t h}$ Eigen value of $D$, and $\lambda_{R}^{(s)}$ the $s^{\text {th }}$ eigenvalue of $R$. Then

$$
\begin{aligned}
& \lambda_{D}=(2-4 r)+2 r \cos \frac{s \pi}{N}, \quad s=1,2, \ldots \ldots . . N-1 \\
& \lambda_{R}^{(s)}=r \\
& \lambda_{s}=(2-4 r)+4 r \cos \frac{s \pi}{N}
\end{aligned}
$$

For such case we can find $\lambda$ by evaluating

i.e.,

$$
\operatorname{det}\left[\begin{array}{cc}
\lambda_{s}-\lambda & -1 \\
1 & -\lambda
\end{array}\right]=0
$$

$$
\lambda=\frac{1}{2}\left(\lambda_{s} \pm \sqrt{-4+\lambda_{s}^{2}}\right)
$$

We want to see under what condition, $\left|\lambda_{ \pm}\right| \leq 1$, two possible cases have been considered.

\section{Case 1:}

If $\lambda_{s}^{2} \leq 4$ then $\lambda$ is complex, then $-1 \leq-r\left(1-\cos \frac{s \pi}{N}\right) \leq 0$

Therefore TTLD scheme is stable for all $0<r \leq \frac{1}{2}$, then the time increment must satisfy the condition $\Delta t \leq \frac{h}{\sqrt{2} c}$.

\section{Case 2:}

If $\lambda_{S}^{2} \geq 4$ then $\lambda$ is real $\left|\lambda_{ \pm}\right|^{2}>1$, In this case the scheme is unstable

\subsubsection{Analytical treatment of consistency}

The convergence of the solution of an approximating set of linear difference equations, (13), to the solution of a linear partial differential equation (10), can be investigated directly by deriving a difference equation for the discretization error e.

Denote the exact solution of the partial differential equation by $E_{\text {exct }}$ and the exact solution of the finite difference equation by $E_{z}$. Then $e=E_{\text {exct }}-E_{z}, \quad$ i.e. $\quad\left(E_{z}\right)_{i, j}^{k}=\left(E_{\text {exct }}\right)_{i, j}^{k}-e_{i, j}^{k}$, $\left(E_{z}\right)_{i, j}^{k+1}=\left(E_{\text {exct }}\right)_{i, j}^{k+1}-e_{i, j}^{k+1}, \ldots \ldots . .$, etc

Substitution into equation (13), and by Taylor's expansion, leads to

$$
\begin{aligned}
e_{i, j}^{k+1}= & r\left[e_{i+1, j}^{k}+e_{i-1, j}^{k}\right]+(2-4 r) e_{i, j}^{k}+r\left[e_{i, j+1}^{k}+e_{i, j-1}^{k}\right]-e_{i, j}^{k-1} \\
& +\frac{\Delta t^{2}}{12}\left(\Delta t^{2} \frac{\partial^{4} E_{e x c t}}{\partial t^{4}}-\frac{h^{2}}{\in \mu}\left(\frac{\partial^{4} E_{e x c t}}{\partial x^{4}}+\frac{\partial^{4} E_{e x c t}}{\partial y^{4}}\right)\right)+\ldots .
\end{aligned}
$$




$$
\begin{aligned}
\left|e_{i, j}^{k+1}\right| \leq & r\left[e_{i+1, j}^{k}+e_{i-1, j}^{k}\right]+(2-4 r) e_{i, j}^{k}+r\left[e_{i, j+1}^{k}+e_{i, j-1}^{k}\right]-e_{i, j}^{k-1} \\
& +\frac{\Delta t^{2}}{12} M\left(\Delta t^{2}-\frac{h^{2}}{\in \mu}\right) \\
\left|e^{k+1}\right| \leq & 2\left|e^{k}\right|-\left|e^{k-1}\right|+\frac{\Delta t^{2}}{12} M\left(\Delta t^{2}-\frac{h^{2}}{\in \mu}\right)
\end{aligned}
$$

Where $\mathrm{M}$ is the modules of the largest of $\frac{\partial^{4} E_{\text {exct }}}{\partial x^{4}}, \frac{\partial^{4} E_{\text {exct }}}{\partial y^{4}}$ and $\frac{\partial^{4} E_{\text {exct }}}{\partial t^{4}}$ and $e^{k}$ and $e^{k-1}$ are the maximum error at $k^{\text {th }}$ and $(k-1)^{\text {th }}$ time-level, respectively then we can prove that

$$
\left|e^{k+1}\right| \leq \frac{1}{2}(k+1)\left(2\left|e^{1}\right|+\frac{k \Delta t^{2}}{12} M\left(\Delta t^{2}-\frac{h^{2}}{\in \mu}\right)\right)-k\left|e^{0}\right|
$$

If the values of $\left(E_{z}\right)_{i, j}^{0}$ and $\left(\frac{\partial E_{z}}{\partial t}\right)_{i, j}^{0}$ are known at initial time, then

$$
\begin{aligned}
& \left(E_{z}\right)_{i, j}^{1}=\left(E_{z}\right)_{i, j}^{0}+\Delta t g_{i, j}^{0}, \text { where }\left(\frac{\partial E_{z}}{\partial t}\right)_{i, j}^{0}=g_{i, j}^{0} \\
& \left(E_{\text {exct }}\right)_{i, j}^{1}=\left(E_{\text {exct }}\right)_{i, j}^{0}+\Delta t\left(\frac{\partial E_{\text {exct }}}{\partial t}\right)_{i, j}^{0}+\frac{\Delta t^{2}}{2 !}\left(\frac{\partial^{2} E_{\text {exct }}}{\partial t^{2}}\right)_{i, j}^{0}+O\left(\Delta t^{4}\right) \\
& \left|e^{1}\right|=\left|E_{\text {exct }}-E_{z}\right| \leq \frac{\Delta t^{2}}{2} M_{i}+\left|e^{0}\right|
\end{aligned}
$$

where

$$
M_{i}=\operatorname{Max}\left[\frac{\partial^{2} E_{\text {exct }}}{\partial t^{2}}+O\left(\Delta t^{2}\right)\right]
$$

then

$$
\left|e^{k+1}\right| \leq \frac{1}{2}(k+1) \Delta t^{2}\left(M_{i}+\frac{k}{12} M\left(\Delta t^{2}-\frac{h^{2}}{\in \mu}\right)\right)+\left|e^{0}\right|
$$

i.e., $E_{z}$ converges to the exact solution as $\Delta t$ and $h$ tends to zero.

\section{Computational Cost Comparisons}

We consider a test case with the following boundary and initial conditions:

$$
\begin{aligned}
& E_{z}(x, y, 0)=\sin (3 \pi x) \sin (4 \pi y) \\
& \frac{\partial}{\partial t} E_{z}(x, y, 0)=0 \\
& E_{z}(0, y, t)=0 \\
& E_{z}(1, y, t)=\sin (3 \pi) \sin (4 \pi y) \cos (5 \pi t) \\
& E_{z}(x, 0, t)=0
\end{aligned}
$$




$$
E_{z}(x, 1, t)=\sin (3 \pi x) \sin (4 \pi) \cos (5 \pi t)
$$

The exact solution in this case is:

$$
E_{z}(x, y, t)=\sin (3 \pi x) \sin (4 \pi y) \cos (5 \pi t)
$$

For the two schemes we choose uniform grid spacing with $\Delta x=\Delta y=h, \Delta t=h^{2}$. The error in $L_{2}$ norm for the two schemes is measured at the same time. The comparison is shown in tables (1:4). The

\begin{tabular}{|c|c|c|c|c|c|}
\hline \multicolumn{3}{|c|}{ two time-level difference Scheme (TTLD) } & \multicolumn{3}{|c|}{ Yee Scheme (FDTD) } \\
\hline$k$ & Max $\|$ error $\|_{2}$ & CPU-time & $k$ & Max $\|$ error $\|_{2}$ & CPU-time \\
\hline 50 & .000499 & 29.06 Seconds & 50 & .000573 & 73.06 Seconds \\
\hline 100 & .00202 & 57.95 & 100 & .00210 & 147.2 \\
\hline 150 & .00452 & 87.7 & 150 & .00461 & 222.454 \\
\hline 200 & .007932 & 116.5 & 200 & .00803 & 289.17 \\
\hline 300 & .0170 & 169.32 & 300 & .0171 & 437.25 \\
\hline
\end{tabular}
programs were written in MATHEMATICA 5.2 and run on IBM PC (160 MHZ).

$$
\begin{gathered}
\text { Max } \| \text { error } \|_{2} \text { at } k^{\text {th }} \text { levels } h=1 / 80, \Delta t=h^{2} \\
E_{z}=\sin (3 \pi x) \sin (4 \pi y) \cos (5 \pi t)
\end{gathered}
$$

\begin{tabular}{|c|c|c|}
\hline Scheme & Max\| $\|$ error $\|_{2}$ & CPU-time \\
\hline two time-level difference Scheme & .02 & 15.2 Seconds \\
\hline Yee Scheme & .02 & 31.1 Seconds \\
\hline
\end{tabular}

Table(2)

at $k^{\text {th }}$ time-level, $k=300, h=1 / 20, \Delta t=1 / 10000$

\begin{tabular}{|c|c|c|}
\hline Scheme & Max $\|$ error $\|_{2}$ & CPU-time \\
\hline two time-level difference Scheme & .01 & 56.2 Seconds \\
\hline Yee Scheme & .01 & 127.1 Seconds \\
\hline \multicolumn{2}{|c|}{ Table(3) }
\end{tabular}

at $k^{\text {th }}$ time-level, $k=300, h=1 / 40, \Delta t=1 / 10000$

\begin{tabular}{|c|c|c|}
\hline Scheme & Max\|error $\|_{2}$ & CPU-time \\
\hline two time-level difference Scheme & .007 & 217 Seconds \\
\hline Yee Scheme & .007 & 488.7 Seconds \\
\hline
\end{tabular}

$$
\text { Table(4) }
$$

at $k^{\text {th }}$ time-level, $k=300, h=1 / 80, \Delta t=1 / 10000$

\section{Conclusion}

The present study shows that the CPU time needed to achieve the same accuracy in Yee scheme is more than two times larger than required for TTLD scheme, this is due to the large computational and storage costs of Yee scheme. 


\section{References}

[1] Amir Yefet and Peter G. Petropoulos. A non-dissipative staggered fourth-order accurate explicit finite difference scheme for the time-domain Maxwell's Equation. Operated by universities space research association. ICASE Report No. 99-30 ,NASA/CR-1999-209514

[2] G. D. Smith. Numerical Solution of Partial Differential Equations: Finite Difference Methods, @ Oxford University Press, 1985.

[3] J. E. Jones, B. Lee. A multigrid method for variable coefficient Maxwell's equations. Siam Journal of Scientific Computing, Volume 27, Pages: 1689 - 1708, 2006

[4] Joe D. Hoffman. Numerical Methods for Engineers and Scientists. Copyright by McGraw-Hill, 1992.

[5] Matt Hansen. Maxwell's Equations, 2004.

http://www.artofproblemsolving.com/LaTeX/Examples/Maxwell's\%20Equations

[6] O. Pironneau. Computer solutions of Maxwell's equations in homogeneous media. Int. J. Numer. Meth. For Fluids Volume 43, Issue 8, Pages 823 - 8382003.

[7] R. Horvath. Application of Numerical Analysis in Computational Science, PhD. course 2004.

[8] Scott Steger. Solving Maxwell's equations in the time domain for arbitrary antenna designs, April 2007, http://www.owlnet.rice.edu/ ssteger/phys416/readme

[9] Stavors V. and Rosemary A. higher-order finite-difference schemes for electromagnetic radiation, scattering, and penetration, part I theory, IEEE Antenna's and propagation magazine, Vol. 44, No. 1, February 2002.

[10] Ulf Andersson. Time Domain Method for Maxwell Equation. Royal Institute of Technology , Stockholm 2001.

[11] Wenhua Yu and Raj Mittra. A conformal FDTD software package modeling Antennas and microstrip circuit component, Antennas and Propagation Magazine, IEEE, Volume: 42, Issue: 5, pages: 28- 39, 2002

[12] Wolfgang Orthurber. A discrete approach to the vacuum Maxwell's equations and the fine structure constant. Arxiv:quant-ph/0312188 vl 23, , 2003

[13] http://en.wikipedia.org/wiki/Maxwell\%27s_equations

[14] http://en.wikipedia.org/wiki/Finite-difference time-domain_method. 